\title{
Prevotella loescheii
}

National Cancer Institute

\section{Source}

National Cancer Institute. Prevotella loescheii. NCI Thesaurus. Code C86672.

A species of obligately anaerobic, Gram-negative, rod shaped bacteria assigned to the phylum Bacteroidetes. This species is nonmotile, non-spore forming, pigmented, fluorescent under UV (360 nm) light, cellobiose, sucrose, lactose, beta-N-acetylglucosaminidase, and alpha-fucosidase positive, but xylose, arabinose, rhamnose, salicin, and glycine aminopeptidase negative. P. loescheii is part of the normal human oral, colonic and vaginal flora, but has been associated with chronic sinusitis, brain abscesses, subdural empyema, septic arthritis, and oral cavity infections. 